\title{
De présidentiable à président : évolution de l'image de François Hollande
}

\author{
Marion Sandré ${ }^{1 *}$ \\ ${ }^{1}$ Laboratoire Babel, Université de Toulon, France
}

\begin{abstract}
Résumé. Cet article propose l'analyse de l'image de l'acteur politique, en croisant les notions d'éthos et de présentation de soi, telles qu'elles sont utilisées en analyse du discours et en analyse des interactions. Il s'agit de montrer comment l'image est construite par les discours de et les discours sur l'acteur politique. Les différentes étapes sont illustrées par le parcours politique de François Hollande, en tant que présidentiable, puis en tant que président. Il s'agit d'analyser dans un premier temps comment il élabore sa propre image, dans et par son discours et par son comportement; et dans un second temps, comment cette image est également construite par d'autres locuteurs, qu'ils soient alliés ou adversaires politiques, instances médiatiques ou citoyennes, qui participent aussi pleinement à l'élaboration de l'image publique de l'acteur politique. Nous verrons plus précisément certains traits importants de l'image de François Hollande, et comment ils se sont développés et ont été négociés par les différents locuteurs: le président «normal», son humour, son respect des citoyens ou encore certaines critiques ou compliments dont il a été l'objet.
\end{abstract}

\begin{abstract}
From candidate to president : Image of François Hollande. In this paper, an analysis of a political actor's image is proposed, based on the notions of ethos and presentation of self, as used in discourse analysis and conversation analysis. The aim is to show that public image is built on discourses of and about the political actor. The political career of François Hollande, from candidate to president, enlights the differents aspects. The analysis focuses first on self- image elaboration, in and by his discourses and behavior. Then, how this image is also built by other locutors, whether they are allies or political opponents, citizen or media organizations : that are also important players in the elaboration of the political actor's public image. More precisely, some of the most important characteristics of François Hollande's image were developped and the way they were handled by differents speakers is analyzed : the "normal » president, his humour, his respects of people, and some negative judgment or compliment about him.
\end{abstract}

\footnotetext{
*marion.sandre@univ-tln.fr
} 


\section{Introduction}

Tout acteur politique, homme ou femme, élu ou non, exerçant au niveau local ou national, façonne de lui une certaine image, au cours de sa carrière professionnelle. Une élection présidentielle est une étape très importante dans un parcours politique, qui met l'acteur sur le devant la scène et l'expose à de nombreux discours, d'abord en tant que présidentiable, puis en tant que président. Je voudrais proposer ici une grille d'analyse permettant d'étudier cette image à partir des nombreux discours qui la façonnent. J'illustrerai cette grille par le parcours politique de François Hollande (désormais FH) en analysant quelques marqueurs de la construction de son image.

Pour mener mon analyse, j'ai construit un très large corpus, à partir des grands discours de la campagne présidentielle (meetings, débats télévisés), des discours officiels (dont les versions vidéos - souvent accompagnées de transcription orthographique - étaient disponibles sur www.elysee.fr), mais aussi d'autres discours médiatiques avec ou parlant de FH. L'objectif n'était pas - bien-sûr - d'être exhaustif, mais de proposer un échantillon choisi des discours qui ont accompagné la campagne présidentielle (2010-2012) et la présidence elle-même (2012-2017). Il s'agit de comprendre comment certains marqueurs de l'image d'un politique sont traités par différents locuteurs et comment ils évoluent nécessairement - dans le temps.

Pour ce faire, avant d'analyser concrètement les discours (2.), je propose de présenter comment l'image d'un président(iable) peut être étudiée (1.).

\section{Analyse de l'image d'un président(iable)}

Le parcours politique d'un président construit de nombreuses facettes. Dans cette première partie, je propose de poser les outils théoriques et méthodologiques permettant d'appréhender son image.

\subsection{Analyser l'image}

Pour analyser l'image d'un acteur politique, il faut d'abord définir ce qu'on entend par « image », puis préciser quels sont les éléments à même de construire cette image.

\subsubsection{Définition de l'image}

Pour analyser l'image de FH, j'utilise la notion d'éthos, soit "l'image que l'orateur donne de lui-même à travers son comportement verbal, sa tenue, son élocution » (Barbéris, 2001 ${ }^{1}$ : 112), telle qu'elle a été développée dans des travaux menés en analyse du discours ${ }^{2}$ et en analyse des interactions verbales ${ }^{3}$, appliquée au discours politique ${ }^{4}$.

Plusieurs de ces approches croisent deux notions :

- l'ethos, emprunté à la tradition rhétorique, qui est un des moyens de persuasion fournis par le discours (avec le pathos et le logos) et qui correspond au " caractère moral de l'orateur qui amène la persuasion, quand le discours est tourné de telle façon que l'orateur inspire la confiance » (Aristote, Rhétorique, I, 2, 4);

- et la notion de «présentation de soi », tirée de la sociologie, qui correspond au comportement social que l'on adopte dans les « interactions sociales » (Goffman, 1959/1973: 23). Si cette théorie ne traite pas proprement du comportement discursif, elle a permis d'ancrer ces questions dans des réflexions interactionnelles et sociales.

En adaptant l'ethos à l'analyse du discours et à de nouveaux corpus, D. Maingueneau propose ainsi de le définir à partir de trois idées principales : 
- L'ethos est une notion discursive, il se construit à travers le discours, ce n'est pas une « image » du locuteur extérieure à la parole ;

- L'ethos est foncièrement lié à un processus interactif d'influence d'autrui ;

- C'est une notion hybride (socio/discursive), un comportement socialement évalué, qui ne peut être appréhendé hors d'une situation de communication précise, intégrée elle-même dans une conjoncture sociohistorique déterminée (2002:60, italique de l'auteur)

Il s'agit donc d'analyser cette image telle qu'elle se construit dans des discours contextualisés et interactifs. Mais si l'ethos est une notion avant tout discursive, il est en lien avec un avant du discours, ce qu'on appelle l' 'éthos prédiscursif » (Maingueneau, 1999), « éthos préalable » (Amossy 1999) ou « réputation » :

Généralement l'orateur n'arrive pas inconnu, mais précédé d'une réputation, qu'il peut éventuellement revendiquer, pour pallier la fragilité des mémoires humaines, et qu'il doit de toutes façons entretenir, en rappelant tant ses hauts faits (vie publique) que ses particularités les plus idiosyncrasiques (vie privée), lorsqu'elles sont propres à servir sa crédibilité. (Constantin de Chanay, $2006: 2$ )

Cette image préalable est particulièrement importante pour les acteurs politiques - $a$ fortiori pour les présidentiables -, dont on connait généralement le parcours et le caractère. Tous ces éléments participent à l'élaboration de cette image, « que chaque énonciation peut confirmer ou infirmer » (Maingueneau, 1999 : 78). Chaque discours déjà proféré par et sur l'acteur politique constitue un socle à l'aune duquel les prochains discours vont être entendus et compris. La construction de l'image est ainsi étroitement liée à la mémoire individuelle et collective. Et elle est partiale et partielle: tous les actes ne sont pas mémorables et mémorés. Cette image ne peut donc être qu'imparfaitement retracée.

\subsubsection{Image et identité}

L'objectif est de mener une analyse de discours prenant en compte un corpus très large (tout type de discours, monologal ou dialogal, médiatique et public), en essayant d'appréhender l'image qui se dégage de ces nombreux discours, soit

l'èthos en un sens plus étendu : force est de constater qu'au fil d'un discours, et de manière analogue, dans la plénitude d'une image, se compose une identité "globale" qui intègre les différentes facettes des différents èthè (préalable ou construit, oratoire ou réel, explicite ou implicite) (Constantin de Chanay, 2006 : $6)$.

C'est cette image que je vais essayer d'analyser ici afin de comprendre comment s'est construite l'identité de FH. En effet,

La question de l'ethos est liée à celle de la construction de l'identité. Chaque prise de parole engage à la fois une prise en compte des représentations que se font l'un de l'autre les partenaires, mais aussi la stratégie de parole d'un locuteur qui oriente le discours de façon à se façonner à travers lui une certaine identité. (Maingueneau, $2002: 58$ )

L'identité discursive est intimement liée à l'identité psychologique et sociale :

Le sujet apparaît au regard de l'autre dans une identité psychologique et sociale qui lui est attribuée, et en même temps se montre à travers l'identité discursive qu'il se construit. Le sens que véhiculent nos paroles dépend à la fois de ce que nous sommes et de ce que nous disons. L'ethos est le résultat de cette double identité, mais qui finit par se fondre en une seule. [...] Identité discursive et identité sociale fusionnent dans l'ethos. (Charaudeau, $2005: 89$ )

Ainsi, en analysant l'ethos, on approche nécessairement l'identité, qui n'est pas une donnée uniquement discursive et qui sort donc du champ de l'analyse du discours ${ }^{5}$. Pour 
autant, on ne peut analyser l'image globale d'un locuteur sans faire référence à son image en tant qu'individu, à son image pré-discursive et à l'évolution de celles-ci durant les sept années que s'étale notre corpus. Mon objectif est néanmoins d'analyser les marqueurs discursifs de l'image, en adoptant une perspective proprement linguistique.

\subsubsection{Les marqueurs de l'image}

Pour analyser l'image d'un président, il faut relever dans les discours les éléments à même de construire cette image :

Se situant quelque part entre le «style » (qui relève davantage de la forme) et l' « identité » (qui relève pleinement du contenu), l'éthos est en tout état de cause un phénomène sémiotique : l'éthos de l'auteur d'un discours quelconque peut être décrit comme une configuration d' "éthèmes » composés de signifiants et de signifiés « éthiques ». (Kerbrat-Orecchioni, $2016: 12$ )

Je vais donc analyser les marqueurs discursifs manifestant une qualité pouvant être mise en relation avec la construction de l'image. Concernant l'image d'un présidentiable puis d'un président, la question est donc de savoir: "quelles sont les qualités d'un "bon président" qui sont éthosisables (c'est-à-dire susceptibles d'être manifestées discursivement) [...] ?» (ibid.: 13). La difficulté de l'analyse de ces marqueurs tient d'abord dans la pluralité de leur nature. Ainsi, il n'existe pas de liste d'éléments porteurs de l'image: ceux-ci sont construits dans chaque discours au regard de la situation d'énonciation.

La difficulté tient également dans la pluralité de leurs significations : ils actualisent des sens très différents.

En ce qui concerne [...] la relation entre signifiants et signifiés éthiques, la difficulté vient de ce qu'il n'y a pas de relation biunivoque entre tel marqueur et telle signification éthique. Tous les marqueurs d'éthos sont ambivalents et inversement, la diversité est extrême des indices possibles pour un même signifié éthique (ibid. : 13)

Les interprétations peuvent être très différentes, et un même marqueur peut produire plusieurs effets (Charaudeau, 2005: 129). On les comprend aussi en fonction de ses convictions politiques : chacun choisit les discours et les actions qu'il veut retenir, ce qu'il veut en retenir, pour élaborer l'image qu'il a envie de construire de l'homme politique. Ainsi, il y a les manœuvres opérées par les différents locuteurs qui participent à l'élaboration de leur image, et il y a la réception de ces marqueurs par l'opinion publique. Les stratégies menées par les acteurs politiques - lorsqu'il s'agit de stratégies conscientes, ce qui n'est pas toujours le cas - ne fonctionnent pas toujours : "l'éthos visé n'est pas nécessairement l'éthos produit » (Maingueneau, 2002 : 59). La construction de l'image est aussi un processus largement involontaire.

Mon corpus présente un grand nombre de discours, au fil desquels l'image évolue. Parce que tous ces discours interagissent entre eux, mon analyse passe nécessairement par la prise en compte des procédés dialogiques, que l'on peut classer en trois catégories (Bres, $\left.2001^{6}: 84\right)$ : les procédés autodialogiques quand le locuteur entre en interaction avec le discours du «je», les procédés dialogiques interlocutif quand le locuteur entre en interaction avec le discours du «tu » et les procédés dialogiques interdiscursifs quand le locuteur entre en interaction avec le discours du « il, elle, on ». Cette répartition permet de classer les différentes références aux discours autres en fonction de leur source énonciative ${ }^{7}$.

Je pointerai donc certains marqueurs discursifs «susceptibles de produire des effets d'éthos » (Charaudeau, $2005: 130$ ). Mais avant cela, je vais aborder la complexité de l'image de l'acteur politique. 


\subsection{La complexité de l'image}

L'image d'un acteur politique est un ensemble complexe, toujours en construction, reposant sur de multiples discours, du fait de l'acteur politique lui-même, et de nombreux autres acteurs sociaux. Il convient donc de donner quelques éléments pour comprendre les différentes caractéristiques de cette image.

\subsubsection{Différents types d'image de l'acteur politique}

L'image d'un homme se construit à des niveaux différents, qui sont intimement liés entre eux. Plusieurs facteurs interviennent : le parcours professionnel, le contexte et, bien sûr, le caractère personnel.

L'image liée au parcours politique : elle naît avec le début de l'engagement politique. Si l'acteur politique n'a pas alors de réputation dans le milieu, il n'arrive pas vierge de toute image: sa région d'origine, sa formation et bien sûr le parti politique dans lequel il s'engage donnent déjà des informations importantes pour appréhender son image ${ }^{8}$.

À partir de l'engagement en politique, chaque étape du parcours professionnel participe à son image. Ces étapes se cristallisent autour de chaque campagne électorale ${ }^{9}-$ dont l'écho est plus ou moins important -, de leur résultat (la victoire ou l'échec participe pleinement à l'image) et des fonctions politiques occupées, qu'il s'agisse d'un mandat électif (lorsque la campagne électorale menée a été victorieuse) ou d'une nomination à un poste. Chaque charge politique concourt à la construction de l'image, même si selon leur portée (locale ou nationale), selon le type de prise de fonction (élection ou nomination), selon leur nombre et leur réussite apparente, les fonctions ont plus ou moins d'importance dans le parcours politique $^{10}$. Il s'agit donc durant ces périodes de faire coïncider son image au "rôle politique $^{11}$ » joué, c'est-à-dire à « l'ensemble des comportements qui sont liés à la position qu'on occupe et qui permettent de faire exister cette position, de la consolider, et surtout de la rendre sensible aux autres » (Lagroye, 1997 : 8). Chaque rôle politique renvoie ainsi un certain nombre de pratiques, notamment discursives, auxquelles l'acteur politique en charge doit se plier.

L'image liée au contexte: d'autres éléments extérieurs influent sur la construction de l'image de l'homme politique. En premier on trouve le contexte socio-culturel du pays en fonction duquel l'acteur politique construit son image. Incarner la politique est différemment considéré selon l'endroit, le régime politique, l'organisation sociale, la situation économique du pays... C'est un contexte général dans lequel certains types d'images sont plus ou moins attendus, espérés ou décriés. Mais il y a aussi le contexte plus réduit, lié aux événements ponctuels, qui permettent la construction d'une image moins sérieuse (grande compétition sportive, par exemple) quand d'autres contraignent à la gravité (attentat, par exemple). Enfin, l'image de l'acteur politique est aussi liée aux genres du discours : selon que le discours est monologal ou dialogal, médiatisé ou non, selon les participants, le cadre spatio-temporel et la finalité du discours, l'image discursive à construire est différente (il doit se montrer plus ou moins sérieux, plus ou moins compétent, plus ou moins pédagogue...). Certains genres du discours sont rattachés à une fonction (les allocutions présidentielles), cette dernière pouvant exclure certains dispositifs (un président - hors campagne pour une réélection - ne débat pas avec ses adversaires). Enfin, le choix des genres, notamment médiatiques, que fait un acteur politique (interview, talk show, l'émission en face à face avec des citoyens, etc.) participe de la construction de son image, en adoptant un certain comportement médiatique plutôt qu'un autre ${ }^{12}$.

L'image personnelle : enfin, l'image de l'acteur politique se construit en relation avec sa personnalité, ses actions et ses engagements personnels. Peuvent également intervenir des éléments concernant son entourage relationnel ou sa vie privée, que ces éléments soient exposés à la demande de l'acteur politique ou contre son gré. Sur ce point, J. Dakhlia 
rappelle qu'il existe une «tradition française de dévoilement stratégique de la vie privée » (2008: 69), mais depuis les années 2000, nous assistons à une nouvelle forme d'« exposition non consentie des représentants politiques » (Desterbecq, $2015: 7$ ). Ainsi, même si elles ne sont pas officiellement affichées, ce sont des informations qui sont souvent dévoilées et qui participent de ce fait à la construction de l'image de l'acteur politique $^{13}$.

Ces éléments renvoient à la fois à l'identité discursive et à l'identité psychologique et sociale. S'ils font pour l'essentiel référence à de l'extra-linguistique, ils sont pourtant intimement liés au linguistique. Ainsi l'ensemble de ces paramètres agissent sur la production des discours: «les thématiques du discours politique sont doublement contraintes : par l'actualité historique et par le statut du locuteur » (Mayaffre, $2012: 85$ ). Et ces différentes influences concourrent à l'élaboration de l'image de l'acteur politique et à son évolution. En effet, l'image n'est pas fixe, elle est sans cesse renouvelée et alimentée par les discours et les actions politiques au cours du parcours politique, en fonction du contexte et en relation avec la personne elle-même. Pour mieux saisir cette évolution, je vais maintenant m'intéresser à deux phases importantes : l'élaboration d'une image de présidentiable, puis de président.

\subsubsection{Une image de présidentiable}

Cette phase correspond à la campagne électorale, d'abord officieuse (période pendant laquelle on teste ses chances) puis officielle. Les discours produits pendant cette période sont particuliers : " la parole qui se déploie en temps de conquête du pouvoir est une parole de promesse » (Charaudeau, $2013: 8$ ). L'objectif est d'être élu, et l'ensemble des discours est dirigé vers cet objectif. Il s'agit pour l'acteur politique de construire de lui même une image de candidat, en « incarn[ant] le rôle du présidentiable » (Théviot, $2016: 52$ ). Pour ce faire, il est nécessaire de développer un éthos de crédibilité et d'identification (Charaudeau, 2005 et 2013) en relation avec le bilan de celui qui est alors encore en fonction : soit dans l'opposition lorsqu'on veut incarner l'alternance, soit dans la continuité s'il s'agit d'un allié politique $-a$ fortiori si on se représente soi-même. Cette phase implique certains types de discours (les meetings par exemple) et d'instaurer avec les citoyens une certaine relation, basée sur une certaine proximité (Le Bart, 2009). Il faut (faire) construire de soi une image de présidentiable, au regard de son parcours politique, du contexte (et notamment du président sortant) et de son propre tempérament.

La campagne de FH n'a pas été simple : «il partait [...] dans la course à 1'Élysée avec un double handicap : pas de légitimité acquise par le fait d'un précédent mandat [...] ; pas de crédibilité quant à une expérience de gouvernant, n'ayant jamais exercé de fonction ministérielle » (Charaudeau, 2013 : 146). Si l'image du candidat est toujours à construire (même s'il s'agit d'une réelection, il faut de nouveau se présenter comme candidat), il est parfois plus ou moins facile d'entrer dans le rôle.

FH est candidat officieux aux primaires socialistes dès novembre 2010 :

«Je ne suis pas dans le calcul des probabilités. Je fais en sorte de pouvoir être candidat. J'ai déjà laissé passer le coup d'avant pour être maintenant dans le coup du jour ! [...] Moi, je n'ai aucune contrainte, je ne suis dans aucun pacte ${ }^{14}$, dans aucun arrangement, si ce n'est dans la loyauté à l'égard de mon parti et dans la responsabilité devant les Français. » (Déclaration à Radio J, le 07/11/2010)

Entre novembre 2010 et mai 2012 - en passant par la déclaration officielle aux primaires citoyennes, le 31/03/2011 -, son objectif est donc de construire de lui l'image d'un présidentiable, à partir de son expérience politique (en tant que conseiller général, maire, député ; premier secrétaire du parti socialiste ; magistrat à la Cour des Comptes) et du contexte (les primaires citoyennes, qui lui octroient la légitimité du corps électoral de gauche; la situation politique du pays, en lien avec la présidence de Nicolas Sarkozy, qui 
l'amène à se positionner contre le monde de la finance ou encore à se présenter comme un futur président «normal»). Sur le plan personnel, FH, connu pour être un homme proche des gens, aimant le consensus et ayant de l'humour, doit adapter son caractère à son rôle de candidat, plus sérieux (Jaubert et Mayaffre, 2013 ; Théviot, 2016).

\subsubsection{Une image de président.}

Cette phase correspond à l'exercice du pouvoir. Il faut alors montrer qu'on est effectivement à la hauteur de la fonction :

"Vous incarnez la France": tels sont les mots que le président du Conseil constitutionnel Jean-Louis Debré adresse à F. Hollande le 15 mai 2012, lors de la cérémonie d'intronisation. La sacralisation du rôle du président de la République est ainsi posée. (Théviot, $2016: 60$ )

Pour façonner une image de président, il faut à la fois prendre en compte l'image idéale du président de la République ${ }^{15}$ et celles des précédents présidents qui ont inévitablement laissé des empreintes à la fonction: "Les candidats doivent donc faire avec cet ethos présidentiel préconstruit, dont les contraintes sont renforcées par les dispositifs communicationnels en vigueur » (Bacot et Gaboriaux, 2016:12). Le nouveau président doit également prendre sa place dans le rôle et y adapter sa propre image.

L'image de président se construit à partir de celle de présidentiable : il existe une « continuité entre le rôle de candidat à la fonction et celui de titulaire de la fonction » (Le Bart, 2009 : 40). Le passage d'une phase à l'autre doit se faire dans un double mouvement de constance et d'ajustement. L'image doit rester cohérente tout en se modifiant :

Chaque éthos se définissant de manière dynamique face aux autres éthè, avec une certaine souplesse d'adaptation, dans les limites toutefois d'une stabilité éthique globale de rigueur (faute de laquelle la crédibilité pâtit d'un déplorable effet de girouette). (Constantin de Chanay, 2009: 63).

Il est important pour un homme politique de ne pas se trahir, mais de montrer aussi une capacité d'adaptation à sa nouvelle fonction. Et ce n'est pas toujours évident :

Il existe un hiatus souvent pointé et autour duquel tourne l'inquiétude démocratique : la (dis)continuité de la parole politique entre le candidat et l'élu, entre les discours de campagne et les discours du trône, entre les promesses électorales et les grand-messes élyséennes. (Mayaffre, $2016: 82$ )

Cette (dis)continuité est tout l'enjeu du passage entre les deux phases. C'est ce qui explique souvent l'incompréhension des citoyens-électeurs qui cherchent dans le discours présidentiel les marques du discours du candidat qui les ont poussés à voter pour lui.

Les discours produits en période de campagne sont pourtant bien différents de ceux produits en période d'exercice : «en temps d'exercice du pouvoir [la parole] est [une parole] de décision" (Charaudeau, 2013: 8). Il faut donc développer une image d'explication et de justification de ses actions politiques. Il ne s'agit plus d'être élu mais d'exercer le mandat, ce qui passe par d'autres genres du discours et d'autres façons d'appréhender la relation interpersonnelle : «le rôle [...] de président, [est] fondé sur [la] symbolique [...] de la grandeur, de la hauteur et du surplomb» (Le Bart, 2009: 39). Il y a un certain nombre de contraintes discursives rattachées à la fonction : «Jouer son rôle de [...] président de la République, c'est s'inscrire dans un sous-ensemble relativement rigide de prescriptions qui encadrent précisément les prises de parole » (ibid., 2003 : 103), depuis les genres du discours à la façon de les pratiquer.

L'image est donc complexe parce qu'elle fait appel à de nombreux actes, de natures différentes, de statuts différents, d'époques différentes, de sources différentes et qu'elle est toujours mouvante, parce que sans cesse nourrie par de nouveaux discours. 


\section{Quelques marqueurs de l'image de François Hollande}

Je vais à présent analyser certains discours qui ont concouru à l'élaboration de l'image de $\mathrm{FH}$, en tant que présidentiable, et en tant que président.

L'image de l'acteur politique n'est pas construite uniquement par lui. L'ensemble des discours tenus par lui et sur lui contribuent à façonner son image :

L'ethos résulte d'une savante alchimie entre ce qui est au fond de l'être, son comportement, son langage et le regard des autres qui dépend lui-même des circonstances dans lesquelles ils perçoivent l'orateur. (Charaudeau, $2013: 106$ )

L'ethos n'est pas uniquement élaborée par le locuteur, qui n'est jamais seul dans sa prise de parole. En appliquant l'interactivité propre aux genres dialogaux à l'interaction générale des discours de l'activité politique, je vais reprendre la dualité proposée par C. Kerbrat-Orecchioni et H. Constantin de Chanay :

En contexte interactif, on parlera d' "éthos en interaction » dans la mesure où l'image projetée (ou affichée) par le locuteur vient se frotter et se confronter à celle qui lui est attribuée par ses partenaires d'interaction, la non-congruence des images projetées et attribuées nécessitant l'intervention de processus « négociatifs » (Constantin de Chanay et Kerbrat-Orecchioni, $2007: 311$ ).

Il y a donc deux grands axes de construction de l'image : l'image affichée par l'acteur politique et l'image attribuée par les autres. Je reprends ces 2 catégories.

\subsection{Image affichée}

L'image affichée est l'image que le locuteur construit de lui-même :

Toute prise de parole implique la construction d'une image de soi. A cet effet, il n'est pas nécessaire que le locuteur trace son portrait, détaille ses qualités ni même qu'il parle explicitement de lui. Son style, ses compétences langagières et encyclopédiques, ses croyances implicites suffisent à donner une représentation de sa personne. Délibérément ou non, le locuteur effectue ainsi dans son discours une présentation de soi. (Amossy, 1999 : 9)

L'acteur politique construit cette image dans toutes ses prises de parole, monologales et dialogales. Une image positive ${ }^{16}$ "dans les limites permises par la "loi de modestie" " (Kerbrat-Orecchioni, 2016:30), qui doit correspondre au parcours politique, au contexte et à l'image personnelle. Pour un président, il s'agit également de mettre en avant des qualités propres à la fonction.

Pour construire l'image, différents élément sont mobilisés : «ce peut être n'importe quel élément (verbal, paraverbal, non verbal) de la matérialité discursive, qui va "manifester" telle ou telle qualité du sujet » (ibid. :12). Un marqueur peut être explicite ou implicite $^{17}$, discursif ou comportemental. Certaines vertus peuvent être dites, d'autres ne peuvent être que montrées, même si «tous les spécialistes de la notion d'éthos insistent sur le fait que les qualités éthiques doivent être "montrées" et non "dites", or toutes les vertus présidentielles ne sont pas discursivement "montrables" " (Kerbrat-Orecchioni, $2016: 13$ ).

On utilisera ici un classement déjà proposé (Sandré, 2014) qui reprend la distinction de Kerbrat-Orecchioni et Constantin de Chanay (2007) en ajoutant ces différentes catégories : l'image affichée dans le discours, par le discours et par le comportement.

\subsubsection{Image affichée dans le discours}

Parfois l'homme politique, dans ses prises de parole, construit de lui explicitement une image qu'il considère comme positive. En effet, certaines qualités qu'on veut mettre en avant passent essentiellement par l'explicitation discursive. C'est le cas du "président normal » proposé par FH au début de sa campagne ou de son opposition au «monde de la 
finance ». Si ces qualités prônées par FH peuvent être montrées (dans le discours et dans les actions) elles peuvent aussi être plus clairement revendiquées.

Je prendrai comme exemple le «président normal », étiquette qui l'a accompagné tout au long de sa campagne et de sa présidence :

Très tôt, il s'est lui-même défini comme un candidat «normal». Qualificatif qu'il n'a pas renié malgré les sarcasmes de ses opposants (y compris à gauche), et sur lequel il est revenu plusieurs fois pour que l'on en comprenne le sens. (Charaudeau, 2013 : 146-147)

C'est ce travail de définition que je vais essayer de retracer ici, en commençant par la première fois que $\mathrm{FH}$ parle de cette caractéristique :

(1)

Journaliste : «Monsieur Hollande, n'êtes-vous pas trop gentil pour un combat comme la présidentielle? Pas assez mordant pour un homme politique ?»

$\mathrm{FH}$ : «Moi, je ne suis pas pour prendre au collet mes adversaires, pour les clouer à un croc de boucher... Est-ce que je suis normal ? Oui. Et je vais vous dire : je pense que le temps d'un président normal est venu » (09/12/2010, déplacement à Alger, en présence de quelques journalistes)

Alors que le journaliste parle d'être « gentil » et « pas assez mordant », FH reformule la question qui lui est posée (en se l'appropriant, comme le montre l'adaptation déictique) en parlant lui, d'être «normal ». L'interrogation est ici un marqueur du dialogisme interlocutif qui permet d'attribuer énonciativement la question à son interlocuteur. La notion de « normalité », proposée par FH, est donc présentée comme discours autre, auquel il donne une réponse affirmative claire.

FH est alors candidat officieux à la primaire citoyenne pour la présidentielle de 2012. Il est crédité de peu de voix et doit se positionner à la fois vis-à-vis de ses concurrents socialistes et de l'actuel président, Nicolas Sarkozy (désormais NS) qui serait son adversaire potentiel à la présidence (Baider, 2015: 126). Les allusions à NS sont très claires : la référence au "croc de boucher» (auquel NS voulait pendre D. de Villepin en 2006, lors de l' " affaire Clearstream ») et la mention de la temporalité à la fin de l'extrait projette une alternance politique qui verrait la non-réélection du président alors en fonction. L'image de l'homme normal ne s'est donc pas construite ex nihilo, elle est très circonstanciée.

Une fois élu candidat officiel du parti socialiste, il redéfinit cette caractéristique dans l'interaction face à NS :

(2)

Laurence Ferrari : «François Hollande, quel président comptez-vous être ? »

FH : « [...] Moi, président d'la république, j'essaierai d'avoir de la hauteur de vue, pour euh fixer les grandes orientations, les grandes impulsions, mais en même temps euh je n'm'occuperai pas de TOUT et j'aurai toujours le souci de la proximité avec les français. J'avais évoqué euh une présidence normale. Rien n'est normal, quand on est président de la république puisque les conditions sont exceptionnelles [...] Bien sûr que le président doit être à la hauteur [...], mais il doit aussi être proche du peuple, être capable de le comprendre » (FH, 02/05/2012, débat de l'entre-deux tours de l'élection présidentielle).

FH propose ici sa conception de la fonction présidentielle, pour répondre à la question de L. Ferrari. L'extrait de la réponse est le dernier moment de la célèbre tirade marquée par l'anaphore «moi, président de la république $»^{18}$. Il revient donc sur ses propres propos (procédé autodialogique) et les précise, à partir de son nouveau statut (finaliste de l'élection présidentielle) mais aussi en fonction des autres discours - critiques et positifs - qui ont été tenus sur lui (voir 2.2.1.) pendant la campagne électorale - qui s'achève alors - et qui participent de la construction de son éthos prédiscursif au moment de l'énonciation. Il maintient sa formule, tout en concédant (par la formule conjonctive «bien sûr que ») la 
nécessité de la hauteur, soit une caractéristique rattachée à la fonction présidentielle (Le Bart, 2009: 39, cité en 1.2.3.). Il ajoute immédiatement (grâce à l'adverbe aussi, qui marque ici l'addition) l'importance de la "proximité », soit une caractéristique rattachée davantage au candidat (ibid., cité en 1.2.2.). Se positionner comme un "président normal» revient alors à faire le lien entre l'image de présidentiable et celle de président.

En tant que président, ensuite, il consolide cette caractéristique, en utilisant d'autres mots :

(3)

FH : «Je resterai ce que je suis, j'ai demandé une présidence qui soit exemplaire, simple, proche mais en même temps je suis pour une présidence d'action et de mouvement » (FH, 09/09/2012, interview sur TF1, « Journal de 20h »)

À la question de C. Chazal sur l'évolution de son implication dans la conduite du gouvernement, FH répond par la constance (les verbes rester et être conjugués au futur et au présent de l'indicatif). Ce qui m'intéresse ici sont les adjectifs qu'il utilise pour qualifier la "présidence». En ce début de quinquennat, la proximité, déjà avancée durant la campagne, est renforcée par l'exemplarité et la simplicité. Ces qualités sont construites par le rôle politique, mais aussi par le contexte (opposition à l' «hyperprésidence » précédente de NS). Les caractéristiques de la présidence normale sont aussi ajoutées (par la formule « en même temps ») à des traits plus attendus de la fonction présidentielle (action et mouvement).

D'autres éléments définitoires sont encore ajoutés deux ans plus tard :

(4)

Thierry Demaizière : « Depuis 2 ans et demi, vos amis, vos ennemis, vos intimes vous critiquent, vous êtes critiqué dans votre vie privée, vous êtes critiqué dans votre vie publique. Est-ce que ça vous touche ? Quelquefois on a l'impression que ça vous touche pas en fait. »

FH : «Je suis un être normal, je l'avais dit. Donc un être normal a un cœur, un esprit, une pensée, des émotions. Mais il se trouve que je suis président de la République. Donc moi je dois garder une forme de pudeur. » (6/11/14, interview sur TF1, «En direct avec les Français »)

(5)

TD : «On ne sait plus exactement qui vous êtes. Y'a 2 ans et demi vous étiez un candidat plutôt euh sympathique et proche des gens et on a l'impression aujourd'hui d'avoir un président insensible euh énigmatique. En quoi le pouvoir vous a changé ?»

FH : «Sympathique, j’espère que je le suis resté. Direct, je pense que je le suis. Proche des Français, je vais le démontrer et je le démontre tous les jours. Sincère, c'est ma conviction. Est-ce que le pouvoir change ? Oui, il change nécessairement $\gg$ (ibid.)

La normalité est ici redéfinie grâce aux concepts de pudeur, d'abord; de sympathie, de proximité et de sincérité, ensuite. En (4), FH reprend son discours (procédé autodialogisme, sous forme de discours rapporté direct) sur l'«être normal», dont il donne d'abord une définition physiologique («être normal» correspond simplement à «être humain »). Mais la fonction présidentielle implique (ce qu'indique l'adverbe de conséquence donc), elle, « une forme de pudeur ». FH justifie par là le fait de ne pas commenter les différents discours sur lui (et notamment concernant sa vie privée), ce qui ne veut pas dire qu'il est insensible (référence à son humanité juste avant). En (5), FH utilise une anaphore de différents adjectifs qui le caractérisent selon lui. Comme dans l'exemple (3), ces qualités sont présentées sous l'axe de la constance (même utilisation des verbes rester et être) et de la répétition («tous les jours »). Il construit ainsi une image stable, notamment depuis son rôle de présidentiable jusqu'à son rôle de président. Mais il concède, en réponse à la question du journaliste qu'il reformule lui-même, avoir «nécessairement » changé, en 
s'adaptant à la fonction présidentielle. Il utilise donc ici des signifiés compatibles à la fois avec son discours de campagne et avec la fonction qu'il incarne.

A la fin de sa présidence, lors de sa visite des bureaux du site Konbini, FH revient sur cette question :

(6)

«j'avais trouvé cette expression, qui a été parfois mal comprise, j’ai dit : “je suis un président normal”. Mais on m'a dit : “qu'est-ce que ça veut dire un président normal ?". Ça veut dire que je n'expose pas nécessairement euh mon être, ma psychologie. J'essaie de faire ce que j'ai à faire. Je suis vraiment président. (06/04/2017, discussion avec la rédaction de Konbini)

Il explique, encore une fois, ce qu'il entend par là. Il reprend son propre discours (phénomène autodialogique, sous forme de discours rapporté direct) et met en scène la demande de définition de l'expression «président normal » par du discours rapporté direct (phénomène dialogique interdiscursif, attribué à un on non défini). La qualité de « normalité », toujours revendiquée, est explicitement définie grâce à la formule « ça veut dire que ». Il reprend ici l'idée de pudeur (la non-exposition de soi) déjà proposée en (4). L'image est donc conservée, mais adaptée à la fonction présidentielle et à l'interprétation qu'en propose $\mathrm{FH}$.

On peut ainsi voir l'évolution d'un élément marquant de sa présidentiabilité et de sa présidence, au travers des discours explicites sur la question.

\subsubsection{Image affichée par le discours}

Dans ses prises de parole, l'homme politique affiche inévitablement une image de lui-même par son discours, de façon implicite. En effet, certaines qualités ne sont pas toujours dicibles (notamment pour des questions de modestie), c'est le cas de l'humour ou le fait d'être un homme de consensus, qui sont des caractéristiques que l'on peut surtout démontrer par ses discours.

Je prendrai ici l'exemple de l'humour, FH étant connu pour avoir de la répartie et savoir faire preuve d'autodérision, même dans des contextes où cela n'est pas forcément attendu. Dans le débat l'opposant à Martine Aubry (désormais MA), FH est ainsi l'auteur de plaisanterie (voir Richard \& Sandré, 2013 : 223).

En tant que président, il a également parfois fait preuve d'autodérision :

(7)

Laurent Delahousse : «Il n’a pas plu aujourd'hui ! C’était magnifique ! »

FH : «C'est exceptionnel! »

LD : (rire) « C'est exceptionnel! »

FH : «Voyez, un président normal peut connaître aussi un jour sans pluie !» (sourire) (14/07/2012, interview sur TF1 et France 2)

$\mathrm{FH}$, pour cette première interview en tant que président, conclut en faisant allusion à l'image du président normal construite pendant la campagne électorale (exemples 1 et 2) et à la météo, qui lui a été particulièrement défavorable dans le premier mois de sa fonction (et par la suite). Ce qui m'intéresse est l'humour dont il peut faire preuve, en répondant à une plaisanterie du journaliste. Il rebondit d'abord en usant d'ironie («c'est exceptionnel !»), entrant ainsi dans le jeu de son interlocuteur. Il reprend ensuite son propre discours de campagne (phénomène autodialogique) et l'adapte à la situation et à l'interaction.

Dans l'exemple suivant, c'est aussi la reprise de son propre discours qui présente une coloration humoristique :

(8)

Maïtena Biraben : «Qui a choisi votre costume aujourd'hui, monsieur le président?» 
FH : « Moi. Moi, président, je choisis mes costumes. » (rire) (19/04/2015, Le supplément sur Canal +)

La question de Maïtena Biraben fait suite à une chronique de Marc Beaugé sur la façon dont FH porte le costume présidentiel (voir exemple 12). FH reprend alors sa fameuse phrase anaphorique du débat de l'entre-deux tours de l'élection présidentielle «moi, président de la république » (voir exemple 2), et l'applique ici au sujet plus trivial proposé par l'émission. Ce phénomène autodialogique est aussi une marque d'autodérision qui inscrit sa pratique des mots d'esprit dans son image de président.

Ces différents exemples participent de la construction d'une image d'un président aimant l'humour, qui est une qualité à doser dans l'exercice de la fonction :

En réalité l'exercice de l'humour en politique s'avère extrêmement contraint par les circonstances liées aux questions de légitimité, de pouvoir effectif, et de place dans l'interaction. (Jaubert et Mayaffre, $2013: 82$ )

Ici, il faut noter l'importance du genre du discours. Pour l'exemple (8), le type d'émission (magazine d'actualité et de divertissement) et le sujet traité s'y prête particulièrement. Mais le genre de l'exemple (7) est plus sérieux : il s'agit cependant de la fin de l'interview, et la plaisanterie est une réponse à celle de l'animateur.

\subsubsection{Image affichée par le comportement verbal :}

Quand il parle, l'homme politique ne produit pas seulement du verbal :

L'ethos, par nature, est un comportement, qui, en tant que tel, articule du verbal et du non verbal pour provoquer chez le destinataire des effets qui ne doivent pas tout aux seuls mots. (Maingueneau, $2002: 59$ )

Ce comportement fait référence aux indices paraverbaux et non verbaux. On peut étendre ce que dit H. Constantin de Chanay pour le débat de l'entre-deux tours à la plupart des discours tenus par les hommes politiques :

Tout du corps est sémiotisé, jusqu'aux indices non communicatifs - rides, sueur, tics, agitation, raideur... - qui ordinairement ne sont pas destinés à être assumés comme ingrédients sémiotiques pertinents dans l'interaction, mais qui en l'occurrence ne manqueront pas d'être portés au crédit ou au discrédit du candidat. (2009: 62)

L'image d'un acteur politique est aussi construite par la façon dont il se comporte dans les discours, monologaux (se montrer un bon orateur) et dialogaux (bon interviewé, bon débattant). Ce comportement doit être concordant avec l'image affichée dans et par le discours, et avec le type d'image qu'on veut construire.

En tant que présidentiable, FH adapte son comportement à la situation. Concernant les débats de l'entre-deux tours : en tant que candidat aux primaires citoyennes, il propose un comportement peu hostile face à Martine Aubry (Richard et Sandré, 2013) alors qu'en tant que candidat à l'élection présidentielle, il est très offensif vis-à-vis de NS (Sandré, 2014).

En tant que président, et au vu des résultats économiques ou face aux attaques terroristes, il se doit d'adopter un comportement grave, un éthos de chef et de solidarité (Charaudeau 2005). Ainsi, l'émotion de son discours du 13/11/2015, alors que les attentats du Bataclan sont encore en cours, est très prégnante. On retrouve cette émotion dans un autre contexte, plus personnel, lors de l'annonce de sa non-candidature à l'élection présidentielle suivante :

(9)

Et aujourd'hui je suis conscient des risques que ferait courir une démarche - la mienne - qui euh ne rassemblerait pas lage- largement autour d'elle. Aussi, j'ai décidé de ne pas être candidat à l'élection présidentielle. Au renouvellement donc de mon mandat (FH, 01/12/2017, allocution à l'Élysée) 
Cette déclaration, au bout de $8 \mathrm{mn} 28$ (pour une durée totale de $9 \mathrm{mn} 55$ ), se fait particulièrement attendre. On remarque la marque d'hésitation euh et le bafouillement sur l'adverbe largement, qui témoignent de l'émotion du candidat au moment le plus important de son discours. Il adopte ici un comportement en harmonie avec le contenu du discours. Si la construction de cette image peut relever d'un processus conscient et calculé du locuteur, elle lui échappe aussi et renvoie à sa façon d'être naturelle. A noter que dans cette déclaration, l'annonce de sa non-candidature est un fait marquant de sa présidence, qui participe également à la construction de son image ${ }^{19}$.

Ainsi, en tant que présidentiable et que président, dans et par les nombreux discours produits par FH, il a lui-même contribué à la construction de sa propre image. Mais il n'est pas le seul à faire cela : sa position amène inévitablement d'autres discours sur lui.

\subsection{L’image attribuée}

L'image attribuée est l'image que les autres locuteurs construisent de l'acteur politique, qu'il s'agisse de partenaires d'interaction dans les discours dialogaux ou de commentateurs de la parole politique dans d'autres discours, qui peuvent vouloir construire de l'acteur politique une image positive ou négative ${ }^{20}$. On parle alors d' «allo-attribution » (KerbratOrecchioni, 2016: 14). Il s'agit de poser des mots sur une attitude jugée positive ou négative : l'image attribuée est souvent une réponse à l'image affichée et il n'est pas rare que l'acteur politique réponde lui-même à cette image attribuée. Les deux images doivent donc être regardées de façon étroite, et on doit prendre en compte dans l'analyse les négociations d'image que les différents discours exposent.

Ces autres locuteurs peuvent être des alliés ou des adversaires politiques, mais aussi des instances médiatiques ou citoyennes. Dans les attendus politiques, les alliés construisent généralement un éthos positif (mais des exceptions sont possibles), les adversaires un éthos négatif (mais là aussi il y a de nombreuses exceptions), les instances médiatiques sont censées être impartiales (mais il y a de nombreuses exceptions, dans un sens comme dans l'autre) et, concernant les instances citoyennes, l'opinion est très variable (perceptible de façon très partielle - et partiale - dans les sondages, les résultats des élections et par le taux de participation aux élections). Cette classification ne doit pas faire oublier que la frontière entre alliés et adversaire est poreuse : le passage de l'un à l'autre est possible ${ }^{21}$, l'inclusion de l'un dans l'autre est possible (on peut trouver des adversaires dans son propre parti ${ }^{22}$ ).

Je vais donc analyser les discours produits sur FH par ces quatre sources énonciatives différentes. De même que pour l'image affichée, l'image attribuée se construit dans et par le discours et par le comportement (Sandré, 2014).

\subsubsection{Image attribuée dans le discours}

Les locuteurs construisent parfois explicitement de l'autre une image.

Il en est ainsi des surnoms, souvent péjoratifs, donnés à FH : Laurent Fabius l'appelait ainsi «monsieur petites blagues » pour faire référence à son humour - considéré comme non adapté à sa position de premier secrétaire du parti socialiste. Arnaud Montebourg l'aurait surnommé «Flamby » en 2003, surnom dont le signifié a été repris par MA en 2011, accusant indirectement FH d'incarner « la gauche molle ». On se souvient également du «capitaine de pédalo » de J.-L. Mélenchon en novembre 2011, critiquant l'absence de fermeté attendue d'un candidat à la présidentielle. Ces différents surnoms alimentent un trait de l'image construite par FH : l'humour - contredisant l'éthos de sérieux attendu - ou le consensus - interprété ici comme une impossibilité à trancher et donc, par extension, à gouverner - affichés par le discours et le comportement. Ces images, qui collent à la peau, peuvent ensuite être reprises et négociées par le principal concerné ${ }^{23}$ ou au contraire être ignorées par lui. 
L'attribution de l'image dans le discours sert également à répondre à l'image affichée dans le discours : les autres locuteurs peuvent reprendre des éléments de langage proposée par l'acteur politique pour lui reprocher. Ce sera le cas du «président normal », qui sera reproché à $\mathrm{FH}$ durant toute la campagne et le quinquennat. Il répondra à ces critiques (voir exemples 2 à 6) dans ses différents discours, qu'il faut aussi interpréter au regard des discours des autres. Durant la campagne, quand FH élabore son image de présidentiable, MA lui reproche déjà cette caractéristique :

(10)

Delahousse : « vous serez une candidate normale? »

MA : «Je suis une femme normale au sens que je vis comme tout le monde, mais je pense que quand on veut être candidat à la présidence de la République, il faut un peu plus » (22/05/2011, interview de 13h sur France 2).

Il ne s'agit pas d'une interaction directe, mais d'une interview de MA par L. Delahousse au début de l'«affaire du Sofitel» concernant D. Strauss-Kahn. Suivant leur accord (voir note 14), MA n'est pas encore officiellement candidate. C'est le journaliste qui fait référence au discours de $\mathrm{FH}$ dans sa question. Il s'agit d'un discours dialogique interdiscursif ne mentionnant pas la source énonciative. Mais à ce moment de la campagne, la formule est déjà entrée dans la mémoire des gens et la référence n'a pas besoin d'être explicitée pour être comprise. L'utilisation de l'adjectif «normale» par MA et donc ici avant tout une reprise interlocutive, qu'elle valide. Elle propose ensuite sa propre définition de la «normalité » (par la formule «au sens où »), immédiatment suivie d'une réserve (introduite par l'adverse adversatif mais) : le signifié de la normalité est présenté de façon négative, comme n'étant pas suffisant. Cette précision lui permet de s'éloigner de la vision de FH, qu'il s'agit clairement de décrédibiliser, pour se poser comme adversaire.

À la fin de la campagne électorale, cette fois-ci en interaction directe, NS critique également cette image :

Oui, Monsieur Hollande, vous avez parlé euh, sans doute pour être désagréable à mon endroit d'un président normal. Je vais vous dire : la fonction d'un président de la république, c'est pas une fonction normale, et la situation qu'nous connaissons, c'est pas une situation normale. Votre normalité, elle n'est pas à la hauteur des enjeux. (NS, 02/05/2012, débat de l'entre-deux tours)

NS reprend le même argument que MA. Il cherche à écorner l'image de $\mathrm{FH}$, en ôtant à la normalité toutes ses caractéristiques positives pour ne conserver que le décalage entre cette proposition et la fonction, dont il peut légitimement parler puisqu'il l'occupe alors. Cette réplique se situe immédiatement après la redéfinition opérée par FH (exemple 2), que NS conteste: à la proximité prônée par FH, NS oppose la « hauteur des enjeux ». Il explicite aussi l'utilisation polémique de cette étiquette (voir exemple 1 et 2), l'accusant d'être « désagréable à son endroit », il s'agit donc clairement de s'opposer à la fois à la caractéristique et à l'utilisation qui en est faite par son adversaire.

Pour finir, et pour contrebalancer avec une image attribuée plutôt positive, pendant la présidence de FH, j'ai choisi un extrait de l'émission Le supplément (cité dans l'exemple 8), dans lequel Marc Beaugé s'adresse à $\mathrm{FH}$, présent sur le plateau :

« Vous portez d'abord des costumes Smuggler, beaucoup moins chers que ceux de vos prédécesseurs, moins de mille euros. Puis vous changez, vous achetez quatre costumes de la créatrice Agnès $b$, une amie et finalement cela ressemble bien à une décision politique. Vous préférez que l'on vous reproche votre dégaine plutôt que vos dépenses. Est-ce que c'est ça aussi être de gauche, monsieur le président ? » (19/04/2015, Le supplément sur Canal +)

Dans cette intervention (intitulé le «bilan stylistique»), sur un ton décalé et humoristique, l'auteur dresse ici l'image d'un président plutôt proche des signifiés positifs 
mis en avant par FH dans sa propre définition de la normalité : « exemplaire, simple et proche » (exemple 3). La comparaison entre FH et ses "prédécesseurs » sert plutôt le président. Le journaliste met en avant la simplicité, la fidélité et l'économie, qualités qu'il ne relie pas à la "normalité », mais au bord politique de FH ("être de gauche »). L'éthos ainsi attribué est plutôt positif et entre en résonance concordante avec l'image affichée par FH dans son discours.

\subsubsection{Image attribuée par le discours}

Les autres locuteurs peuvent également construire l'image de l'autre de façon plus indirecte. L'objectif peut être de critiquer l'acteur politique pour lui contester des caractéristiques favorables. C'est ce que MA fait ici en lui reprochant deux choses : son inexpérience et son profil de « rassembleur », que FH vient d'afficher dans son discours :

(13)

DP : «c'est un handicap pour François Hollande de ne jamais l'avoir été [ministre] ?»

MA : « Je dis mes avantages. Voyez on parlait tout à l'heure dans le fond du contrat génération, et je le dis en toute amitié à François : ça ne marche pas. Et c'est là où l'expérience a un sens parce qu'on l'a fait, on sait qu'ça marche pas. J'écoute les syndicats, je sais qu'ils ont aussi raison, et je crois réellement que l'expérience est nécessaire. François parle beaucoup du rassemblement depuis deux trois jours. Moi j'ai trouvé un parti socialiste qui n'était pas rassemblé. Et j'ai mis trois ans, ça n'a pas été facile et j'peux vous dire que la première année m'a même rendue très triste » $(12 / 10 / 2011$, débat de l'entre-deux tours de la primaire citoyenne)

MA est à la fois une adversaire et une alliée de $\mathrm{FH}$, cette interaction est construite autour de ces deux tensions (Richard \& Sandré 2013). Ici, c'est clairement l'opposition qui domine, sous un vernis d'entente affichée (« en toute amitié », utilisation du prénom) : MA accuse son concurrent de n'avoir pas d'expérience (contrairement à elle, son image affichée par le discours se construit en miroir) et d'avoir divisé leur parti (alors qu'elle, elle l'aurait rassemblé, c'est donc la même stratégie). Or l'expérience et la qualité de rassembleur sont des qualités très importantes pour un candidat à l'élection présidentielle. MA utilise donc le parcours politique de son adversaire pour construire de lui l'image d'un candidat qui n'est pas à la hauteur. Cette même stratégie de critique a été utilisée par tous les adversaires politiques de FH : voir par exemple les critiques de NS sur la compétence, l'honnêteté ou le sérieux de FH lors du débat de l'entre-deux tours (Sandré 2014 : 80-82).

L'objectif peut également être de donner de l'acteur politique une image positive. Ainsi, en mai 2017, le journaliste Yann Barthès fait le bilan du quinquennat après avoir diffusé un reportage sur les « moments qui nous ont fait le plus rigoler » :

(14)

«En tout cas, sachez que pendant le quinquennat de FH, nos équipes ont toujours été respectées par l'Elysée, elles ont toujours été traitées à égalité avec les autres équipes de télévision, François Hollande nous a parlé quand on avait des questions à poser sur des sujets difficiles, nos équipes ont toujours été libres de travailler avec la présidence, libres de le critiquer, libres de se moquer d'lui, comme on vient de le faire pendant 5 minutes. Sans un seul coup de fil. Pas un coup de fil. » (12/05/2017, Émission Quotidien, TMC)

Le journaliste de l'émission d'infodivertissement peint du président une image plutôt respectueuse des journalistes et de la liberté d'expression, ce qui contraste avec la façon dont certaines personnalités politiques traitent les journalistes de cette émission ${ }^{24}$. Il s'agit donc de construire, par son discours, une image positive. Ici, plus que le genre du discours, c'est le moment qui rend cet hommage possible : c'est un bilan à l'issue du quinquennat. 


\subsubsection{Image attribuée par le comportement}

Enfin, les autres locuteurs peuvent, par leur comportement, contribuer à construire d'un acteur politique une certaine image. Ces marqueurs sont plus difficiles à appréhender.

Lors de sa prestation dans la matinale de France Inter, le 05/01/2015, FH a été beaucoup interrompu par le présentateur, Patrick Cohen et par les différents chroniqueurs (notamment Thomas Legrand). Par ce comportement offensif, les journalistes construisent de leur invité une certaine image. Certains diront que le président est affaibli et qu'il ne parvient plus à imposer le respect dans un échange; d'autres diront que le président n'a pas peur du dialogue et qu'il interprète différemment la fonction présidentielle (être plus «proche et simple »). De façon plus large, la parole présidentielle est aujourd'hui associée à de nouveaux genres, et de principalement monologale, elle devient de plus en plus dialogale. La présence d'interruptions de parole est alors inévitablement liée à ces nouvelles configurations discursives.

Autre exemple, contribuant à l'image de FH est l'ovation qu'il a reçue à la fin de son discours, suite aux attentats de Paris :

(15) Le terrorisme ne détruira pas la République. Car c'est la République qui le détruira. Vive la République et vive la France. (16/11/2015, Congrès à Versailles)

Tout le congrès s'est alors levé et a applaudi longuement le président de la République, en chantant la Marseillaise. Le comportement de tous les députés et sénateurs, alliés et adversaires, dans ce moment particulièrement important suite aux événements graves contribuent à présidentialiser FH et à construire de lui l'image d'un président rassembleur et fort. Toutefois, cet épisode est très circonstancié : le comportement du congrès n'est pas uniquement une réaction au discours du Président, mais également aux événements (lien avec le contexte ponctuel). Le respect et la cohésion ainsi témoignés sont autant tournés vers les institutions et la fonction présidentielle que la personne de FH. C'est pourtant son image qui bénéficie de ce moment.

Ainsi, l'acteur politique n'est pas le seul à parler, les autres locuteurs coucourent également à construire de lui une certaine image : "l'identité n'est rien d'autre qu'une construction interactive, que chacun se compose en "composant" avec autrui » (KerbratOrecchioni, 2002 : 187, italique de l'auteur). En effet, comme FH le répond lui-même à la question d'un journaliste lui demandant comment il ressent les choses, le matin du second tour de l'élection présidentielle de 2017: "Pour être aimé faut être deux» (FH, 07/05/2017). Un homme politique ne peut construire son image seul. Celle-ci est une partition qui fait intervenir plusieurs voix.

\section{Conclusion}

L'image d'un acteur politique est multiple et complexe : elle se construit à partir de plusieurs sources, elle est de plusieurs natures et revêt plusieurs significations. Chaque discours de et sur lui apporte sa pièce à l'édifice de l'image. Celle-ci n'est jamais donnée, elle est sans cesse travaillée, actualisée, renouvelée.

Les étapes de la campagne électorale puis de l'exercice du pouvoir sont des moments forts dans la construction de l'image d'un politique. La question de la (dis)continuité entre ces deux phases est au cœur de cette analyse. J'ai voulu montrer comment différents marqueurs d'ethos se manifestaient dans les discours et les comportements et l'évolution de ces marqueurs au cours des sept années du corpus.

L'image de FH a ainsi évolué depuis sa carrière politique, son statut de présidentiable jusqu'à son image de président. J'ai abordé plusieurs marqueurs de son image, explicites (marqueurs construits dans le discours) ou plus implicites (marqueurs construits par le 
discours et par le comportement), qui ont fait l'objet de nombreux discours à la fois par FH et par d'autres locuteurs (alliés, adversaires, instances médiatiques ou citoyennes). J'ai ainsi montré comment l'image du "président normal » avait traversé la campagne et comment elle s'était adaptée à l'exercice du pouvoir; ou comment l'image d'un homme « rassembleur » ou « drôle » avait pu être traitée au fil du temps. L'objectif d'un politique est de rester fidèle à son image - une certaine image qu'il se fait de lui, qu'on se fait de lui - en l'adaptant nécessairement à la fonction politique occupée (candidat ou président) et au contexte. Les différents marqueurs de son image sont ensuite (ré)interprétés différemment selon les personnes, au regard de leurs propres convictions politiques. Enfin, l'image d'un président est, suite à son exercice du pouvoir, relativisée par la présidence suivante et l'opinion publique retient certains éléments plutôt que d'autres dans l'ensemble des discours qui font un président.

\section{Références bibliographiques}

Amossy R. (1999). «L'Ethos au carrefour des disciplines : rhétorique, pragmatique, sociologie des champs ", dans R. Amossy (éd.), Images de soi dans le discours. La construction de l'ethos. Lausanne : Delachaux et Niestlé, p. 129-156.

Amossy R. (2000/2013). L'Argumentation dans le discours. Paris : Nathan/Armand Colin.

Amossy R. (2010). La présentation de soi. Ethos et identité verbale, Paris : PUF.

Amossy R. (2014). «L'éthos et ses doubles contemporains. Perspectives disciplinaires », Langage et société, $\mathrm{n}^{\circ} 149$, Ethos discursif, p. 13-30.

Amossy R. (éd.) (1999). Images de soi dans le discours. La construction de l'ethos, Lausanne: Delachaux et Niestlé.

Aristote, Rhétorique, traduction française P. Chiron (2007), Paris : Flammarion.

Bacot P. et Gaboriaux C. (2016). « Discourir pour présider ». Mots. Les langages du politique, $\mathrm{n}^{\circ} 112$, Discours présidentiels et de présidentielles, p.9-17.

Baider F. (2015). «Évaluation de la présidentiabilité (à l'interface de la sémantique lexicale et de la linguistique de corpus) ». Mots. Les langages du politique, $\mathrm{n}^{\circ} 108$, Thèmes et thématiques dans le discours politique, p. 103-128.

Cabasino F. (2009). «La construction de l'ethos présidentiel dans le débat télévisé français ». Mots. Les langages du politique, ${ }^{\circ}$ 89, 2007. Débats pour l'Élysée, p. 11-23.

Chabrol C. (2006). " "Identités" Sociales et discursives ». Questions de communication, $\mathrm{n}^{\circ}$ 9, Rôles et identités dans les interactions conflictuelles, p. 5-27.

Charaudeau P. (2005). Le Discours politique. Paris : Vuibert.

Charaudeau P. (2013). La conquête de pouvoir. Opinion, persuasion, valeur. Les discours d'une nouvelle donne politique. Paris : L'Harmattan.

Charaudeau P. (2015). « Le charisme comme condition du leadership politique ». Revue française des sciences de l'information et de la communication, $\mathrm{n}^{\circ}$ 7, http://rfsic.revues.org/1597.

Constantin de Chanay H. (2006). "Pouvoir des images d'avant le pouvoir: de l'ethos dans les portraits des candidats à l'élection présidentielle 2002 en France ». Sémiotica, n 159, 1/4, p. 151177.

Constantin de Chanay H. (2009). « Corps à corps en 2007 : Nicolas Sarkozy face à Ségolène Royal ». Itinéraires. Littérature, Textes, Cultures, ${ }^{\circ} 1$, p. 61-80.

Constantin de Chanay H. et Kerbrat-Orecchioni C. (2007), « 100 minutes pour convaincre : l'ethos en action de Nicolas Sarkozy », dans M. Broth et al. (éds), Actes du colloque Le Français parlé des médias. Stockholm : Acta Universitatis Stokholmiens, p. 309-329.

Dakhlia J. (2008). « La représentation politique à l'épreuve du people : élus, médias et peopolisation en France dans les années 2000 ». Le Temps des médias, n 10, Peopolisation et politique, p. 6681.

Desterbecq J. (2015). La peopolisation politique. Louvain-la Neuve : De Boeck.

Détrie C. et al. (éds) (2001). Termes et concepts pour l'analyse du discours, Paris: Honoré Champion.

Goffman E. (1959/1973). La Mise en scène de la vie quotidienne, tome 1, La Présentation de soi, Paris : Minuit. 
Jaubert A. et Mayaffre D. (2013). «Ethos préalable et éthos (re)construit. La transformation de 1'humour légendaire de François Holande ». Langage et société, ${ }^{\circ}$ 146, p. 71-88.

Kerbrat-Orecchioni C. (2002). «Rhétorique et interaction », dans R. Koren et R. Amossy (éds), Après Perelman: quelles nouvelles politiques pour les nouvelles rhétoriques?. Paris: L'Harmattan, p. 173-196.

Kerbrat-Orecchioni C. (2016). «Les débats présidentiels comme lieu de confrontation d'éthos : une approche interactionnelle du discours politique », dans M. Degani, P. Frassi et M.I. Lorenzetti (éds). The Languages of Politics, vol. 2, Cambridge Scholars Publishing, p. 9-32.

Kerbrat-Orecchioni C. (2017). Les débats de l'entre-deux-tours des élections présidentielles françaises. Paris : L'Harmattan.

Lagroye J. (1997). «On ne subit pas son rôle ». Politix, n 38, L'institution des rôles politiques, p. 717.

Le Bart Ch. (2003). «L'analyse du discours politique : de la théorie des champs à la sociologie de la grandeur ». Mots. Les langage du politique, $\mathrm{n}^{\circ} 72$, p. 97-110.

Le Bart Ch. (2009). «Les présidentiables de 2007 entre proximité et surplomb ». Mots. Les langages du politique, ${ }^{\circ} 89,2007$. Débats pour l'Élysée, p. 39-55.

Maingueneau D. (1998). Analyser les textes de communication. Paris : Dunod.

Maingueneau D. (1999). «Ethos, scénographie, incorporation ». dans R. Amossy (éd.), Images de soi dans le discours. La construction de l'ethos, Lausanne, Delachaux et Niestlé, p. 75-102.

Maingueneau D. (2002). «Problèmes d'ethos ». Pratiques, n 113-114, p. 55-67.

Maingueneau D. (2014). «Retour critique sur l'ethos ». Langage et société, $\mathrm{n}^{\circ} 149$, Ethos discursif, p. 31-48.

Mayaffre D. (2012). Mesure et démesure du discours. Paris : Presses de Sciences-Po.

Mayaffre D. (2016). «Du candidat au président. Panorama logométrique de François Hollande». Mots. Les langages du politique, $\mathrm{n}^{\circ} 112, \mathrm{p} .81-92$.

POLITIX, $\mathrm{n}^{\circ} 28,(1994)$, Le métier d'élu : jeux de rôles.

POLITIX, $\mathrm{n}^{\circ} 38$, (1997), L'institution des rôles politiques.

Richard A. et Sandré M. (2013). «Le débat télévisé de primaires : un nouveau genre médiatique de discours politique en France ». Nottingham French Studies, vol. 52, $\mathrm{n}^{\circ} 2$, Vivement dimanche: Media, Politics and France's Electoral Year 2011-12, p. 215-226.

Sandré M. (2011). «Dialogisme, comportement et débat politique télévisé : Ségolène Royal lors du débat de l'entre-deux tours ». dans A. Jaubert et al. (dir.), Discours rapporté, citation et pratiques sémiotiques, volume 2, Citations II. Citer pour quoi faire? Pragmatique de la citation, Academia Bruylant, p. 241-256.

Sandré M. (2014). «Ethos et interaction : analyse du débat politique Hollande/Sarkozy ». Langage et société, n 149 , Ethos discursif, p. 69-84.

Théviot A. (2016). « 'Rôle' politique et modelage des identités de genre. Du candidat au Président : mise en scène virtuelle des masculinités de François Hollande ». Genre en séries, n 3, p. 45-74.

Weizman E. (2006), "Rôles et identités dans les interactions conflictuelles». Questions de communication, $\mathrm{n}^{\circ}$ 9, p.7-13.

Weizman E. (dir.) (2006). Questions de communication, $\mathrm{n}^{\circ}$ 9, Rôles et identités dans les interactions conflictuelles.

${ }^{1}$ Termes et concepts pour l'analyse du discours, Détrie et al. (éds), entrée « Ethos ».

${ }^{2}$ Qui a fait l'objet de nombreuses publications. Voir notamment : Amossy (éd.), 1999 ; Amossy, 1999, 2000/2013, 2010, 2014 ; Maingueneau, 1998, 1999, 2002, 2014.

${ }^{3}$ L'approche interactive de la notion d'ethos a surtout été développée par Catherine KerbratOrecchioni et Hugues Constantin de Chanay: Constantin de Chanay, 2006, 2009; Constantin de Chanay et Kerbrat-Orecchioni, 2007 ; Kerbrat-Orecchioni, 2002, 2016, 2017 ; voir aussi Sandré, 2014.

${ }^{4}$ Charaudeau, 2005, 2013 et 2015.

${ }^{5}$ Concernant l'identité et les approches croisées en analyse du discours et en psychologie sociale, voir Weizman (dir), 2006 et plus précisément Weizman, 2006 et Chabrol, 2006.

${ }^{6}$ Bres, $2001: 84$, dans Termes et concepts pour l'analyse du discours, Détrie et al. (éds), entrée « Dialogisme».

${ }^{7}$ Sandré, 2011.

${ }^{8}$ Ainsi, FH, lorsqu'il devient président du comité de soutien de François Mitterand en 1974 est un militant de l'UNEF (Union Nationale des Étudiants de France, organisation étudiante, classée plutôt à 
gauche), étudiant d'HEC (École des Hautes Études Commerciales) et de l'IEP (Institut d'Études Politiques, surnommé « Sciences Po ») Paris. Il adhère ensuite au Parti Socialiste (désormais PS) en $1979^{8}$ et sort diplômé de l'ENA (École Nationale d'Administration) en 1980 (promotion Voltaire). Ces éléments construisent déjà de l'homme une certaine image, en lien avec sa formation et son bord politique.

${ }^{9}$ Au cours de laquelle il faut montrer qu'on peut être à la hauteur de la fonction convoitée : auprès des électeurs de son propre parti (élection à la tête du parti, élection aux primaires pour les élections présidentielles), auprès des grands électeurs (élection au suffrage universel indirect) ou auprès des citoyens-électeurs (élection au suffrage universel direct).

${ }^{10} \mathrm{FH}$ a ainsi mené des campagnes électorales malheureuses $(1981,1993)$ et d'autres victorieuses : à la députation en 1988, 1997 ; à la tête du Parti Socialiste en 1997 ; à la mairie de Tulle en 2001 ; au conseil général de Corrèze en 2008; aux Primaires citoyennes en 2011 et à la présidence de la république en 2012 (http://www.elysee.fr/la-presidence/francois-hollande-biographie-2/).

${ }^{11}$ Sur la notion de « rôle politique », voir les numéros 28 (1994) et 38 (1997) de la revue Politix.

${ }^{12} \mathrm{FH}$ participe aux genres traditionnellement rattachés aux fonctions de candidats à la présidence et de président (débat de l'entre-deux tours, vœux aux Français, allocution du 14 juillet) et à d'autres genres plus nouveaux : débat de l'entre-deux tours d'une primaire interne à un parti politique, face à face avec des Français ou des émissions plus divertissantes, comme l'émission radiophonique $L$ 'œil du tigre, présentée par Philippe Colin, sur France Inter, le 05/06/2016, dont FH dit, après avoir été présenté : «merci à vous de m'avoir invité ici dans L'œil du tigre, c'est pas si fréquent. »

${ }^{13}$ Pour FH, certains éléments de sa vie privée ont été couverts par les médias : vie maritale avec Ségolène Royal, médiatisation de la naissance de leur quatrième enfant, ainsi que sa relation avec Valérie Trierweiler jusqu'à leur rupture et la publication du livre de cette dernière Merci pour ce moment, en 2014. La révélation de sa liaison avec Julie Gayet par un magazine people en janvier 2014 a également été particulièrement commentée.

${ }^{14} \mathrm{FH}$ fait ici référence au pacte qui aurait été scellé entre Martine Aubry et Dominique StraussKahn de ne pas se présenter l'un contre l'autre. En Mai 2011, suite à l'«affaire du Sofitel », le second doit renoncer à se présenter aux primaires citoyennes, ce qui permet à M. Aubry d'être candidate.

${ }^{15} \mathrm{C}$ 'est-à-dire « une image proche du rôle présidentiel et coïncidant avec les représentations que se font les Français du chef de l'État à l'époque contemporaine $[\ldots] »$. Pour construire cette image il faut donc « tenir compte des attentes de l'imaginaire collectif.» (Cabasino, 2009 : 22).

${ }^{16} \mathrm{Il}$ est à noter que certains discours de FH l'ont particulièrement desservi : voir son discours sur l'«affaire Léonarda » (19.10.13) ou sur la déchéance de nationalité française des binationaux (16.11.2015). Enfin, le livre « Un Président ne devrait pas dire ça... » (Lhomme et Davet, 12 octobre 2016), qui retranscrit les confidences du président à deux journalistes, a également participé à l'élaboration d'une image plutôt négative du président.

${ }^{17}$ Voir la distinction proposée par D. Maingueneau entre l'éthos «dit» et l'éthos «montré » (2002: 65).

${ }^{18}$ L'ensemble de cette tirade s'oppose en creux à NS (Sandré, 2014: 75) : le «moi, je » se présente comme une forme d' " auto-attribution explicite » couplée à une «allo-attribution implicite » (Kerbrat-Orecchioni, $2016: 19$ ).

${ }^{19}$ En témoigne par exemple l'échange suivant, tiré de la discussion avec la rédaction de Konbini (06/04/2017), déjà mentionnée dans l'exemple 6 :

Journaliste : «Ce que je retiens de vous c'est que j'ai l'impression que vous êtes très humain»

$\mathrm{FH}:$ «ça je vous le confirme » (rires)

Journaliste : «Et bizarrement ce qui m'a le plus confirmé ça, c'est quand vous avez décidé de ne pas vous représenter »

FH : « et ce choix-là qu'j'ai fait, je pensais que c'est celui qui permettait d'avoir un regard différent sur ce que j'avais fait. J'ai pas l'obsession du pouvoir ».

${ }^{20}$ « sans toutefois tomber dans l'injure, ce qui risquerait d'être contre-productif : c'est ce que l'on peut appeler l'“effet boomerang". (Kerbrat-Orecchioni, $2016: 30$ ). Voir pourtant les insultes proférées par NS pendant le débat (Sandré 2014).

${ }^{21}$ Ainsi, J.-L. Mélenchon était socialiste, finaliste perdant contre FH en 1997 à la tête du parti. En 2008, il a quitté le parti et s'est positionné durant le quinquennat dans l'opposition en marquant fortement la distance entre son ancien et son nouveau parti. 
${ }^{22}$ MA est une alliée officielle, mais adversaire lors des primaires citoyennes et adversaire officieuse, proche des frondeurs, dans la conduite de la politique du quinquennat.

${ }^{23}$ En témoignent ces deux exemples, faisant écho à sa «mollesse » présumée. Le premier est un échange informel, lors d'un déplacement à Amiens (11/05/2012)

Femme : «Vous avez une poignée de mains vigoureuse » (rire)

$\mathrm{FH}:$ : ouais »

Homme : «Elle est pas molle»

Autre homme : « La main » (rires)

$\mathrm{FH}$ : «Ni la main ni la tête (rires) je vous dis rien du reste ! »

Le second est un discours officiel, le 28/04/2016, lorsqu'il reçoit les auteurs de l'ouvrage Football de légendes, une histoire européenne à l'Élysée : «Parfois les mous peuvent atteindre la perfection, la subtilité, l'élégance, la surprise ».

Dans ces deux exemples, FH reprend la critique à son avantage, soit en réponse, soit à son initiative, en usant d'humour.

${ }^{24}$ Notamment Marine Le Pen et Jean-Luc Mélenchon. 